\title{
THE MECHANISM OF FORMATION OF MICROPOROUS OR SKINNED MEMBRANES PRODUCED BY IMMERSION PRECIPITATION
}

\author{
J.G. WIJMANS, J.P.B. BAAIJ and C.A. SMOLDERS \\ Department of Chemical Technology, Twente University of Technology, Enschede \\ (The Netherlands)
}

(Recieved July 6, 1982; accepted November 2, 1982)

\section{Summary}

Cellulose acetate and polysulfone casting solutions were coagulated in water/solvent mixtures with differing solvent content. Precipitation in pure water yielded skinned membranes. Precipitation in water/solvent mixtures with solvent concentration exceeding a certain minimum value (which is different for different systems) resulted in microporous membranes. This phenomenon has been explained in terms of the model description for the formation of asymmetric membranes as adopted in our laboratory. In this model, the skin formation is related to gelation and the formation of the porous substructure to liquid-liquid phase separation.

It is made plausible that the addition of solvent to the coagulation bath favours nonsolvent inflow and hence liquid-liquid demixing in the precipitating film.

\section{Introduction}

The majority of commercially available synthetic membranes are produced by the so-called phase inversion process. In this process, a homogeneous polymer solution is brought to phase separation by means of a penetrating nonsolvent and/or a solvent outflow.

The concept "phase inversion process" covers a range of different techniques, each leading to a specific membrane structure. The two techniques that will be discussed in this paper are (i) precipitation from the vapour phase, and (ii) immersion precipitation. Precipitation from the vapour phase yields symmetric microporous membranes whereas the immersion precipitation process usually results in asymmetric ultra- or hyperfiltration membranes. These membranes are called asymmetric since their cross-section reveals an asymmetric structure: a very dense and thin skin supported by a porous sublayer.

Recently it was discovered in our laboratory [1] that with immersion precipitation it is also possible to obtain microporous membranes; to achieve this, solvent was added to the coagulation bath. This is a very interesting phenomenon since knowledge about ways to avoid the formation of a skin may lead to a better understanding of skin formation. 
Examples of this type of membrane formation for various polymers will be given and discussed in terms of the formation mechanism, especially considering how the addition of solvent to the coagulation bath prevents skin formation.

\section{Theory}

\section{Phase separation}

Several authors have tried to explain the formation of asymmetric membranes in terms of phase separation phenomena in polymer solutions [2-9]. In recent years, experimental evidence was gathered in our laboratory $[6,7$, $10,11]$ that two different types of phase separation phenomena are responsible for the asymmetric structure of the membranes.

In a system consisting of a polymer, a solvent and a nonsolvent, the demixing types that can be distinguished are (see Fig. 1):

(i) Liquid-liquid phase separation:

The solution lowers its free enthalpy by separating into two liquid equilibrium phases. There are two ways for this demixing to occur: by nucleation and growth of droplets of the second phase and by instantaneous spinodal demixing. The spinodal demixing gap is surrounded by the composition area where phase separation by nucleation and growth takes place; since this latter phenomenon is a relatively fast process, spinodal demixing is not very probable in polymer solutions.

(ii) Crystallization or gelation:

The polymer molecules decrease the free enthalpy of the solution by forming ordered structures. At low polymer concentrations single crystals will be formed, but at higher concentrations the usually very small crystalline regions can act as physical crosslinks between the polymer molecules and the result is a thermoreversible gel.

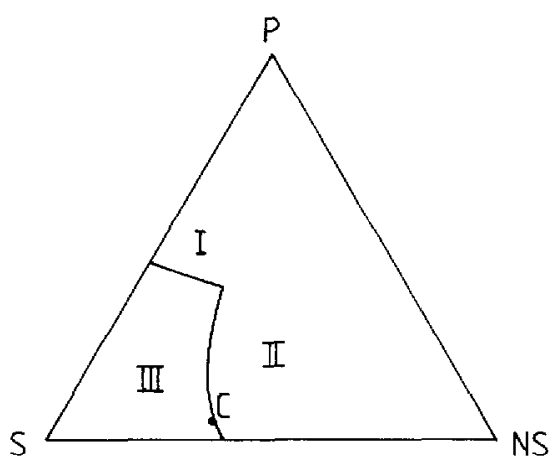

Fig. 1. Ternary phase diagram; P : polymer; $\mathbf{S}$ : solvent; NS : nonsolvent; C: critical point; I: gelation; II: liquid-liquid phase separation; III: homogeneous solution. 


\section{Mechanism of formation}

In the immersion precipitation process a polymer solution, cast on a support, is immersed in a bath containing a nonsolvent. As was first suggested by Koenhen [6], we assume that the skin is formed by gelation and that the porous sublayer is the result of liquid-liquid phase separation by nucleation and growth. The factor determining the type of phase separation at any point in the cast film is the local polymer concentration at the moment of precipitation. In the first split second after immersion there is a rapid depletion of solvent from the film and a relatively small penetration of nonsolvent. This means that the polymer concentration at the film/bath interface increases and that the gel boundary is crossed (transition III to I in Fig. 1). The thin and dense gel-layer that is formed in this way, the skin, will act as a resistance to solvent outdiffusion, and at positions beneath the toplayer, demixing will occur at lower polymer and higher nonsolvent concentrations. So here the type of demixing will be liquid-liquid phase separation (transition III to II in Fig. 1). The demixing gap is entered at the polymer-rich side of the critical point, so the nuclei consist of the polymer-poor phase, and the result is a porous structure, the pores of which are filled with the dilute solvent/nonsolvent phase.

The approximate changes of composition for the toplayer and the substructure, the so-called "coagulation paths", are given in Fig. 2. A more detailed description of the concentration profiles in a precipitating casting solution can be found in the paper by Bokhorst et al. [9] .

In the case of precipitation from the vapour phase, the cast polymer solution is in contact with a nonsolvent vapour phase, saturated with the solvent used. In this process there is no solvent outflow but only a nonsolvent inflow. The change of composition in the film is illustrated in Fig. 3: the only possible demixing mechanism is liquid-liquid phase separation and the result is a microporous membrane without a skin.
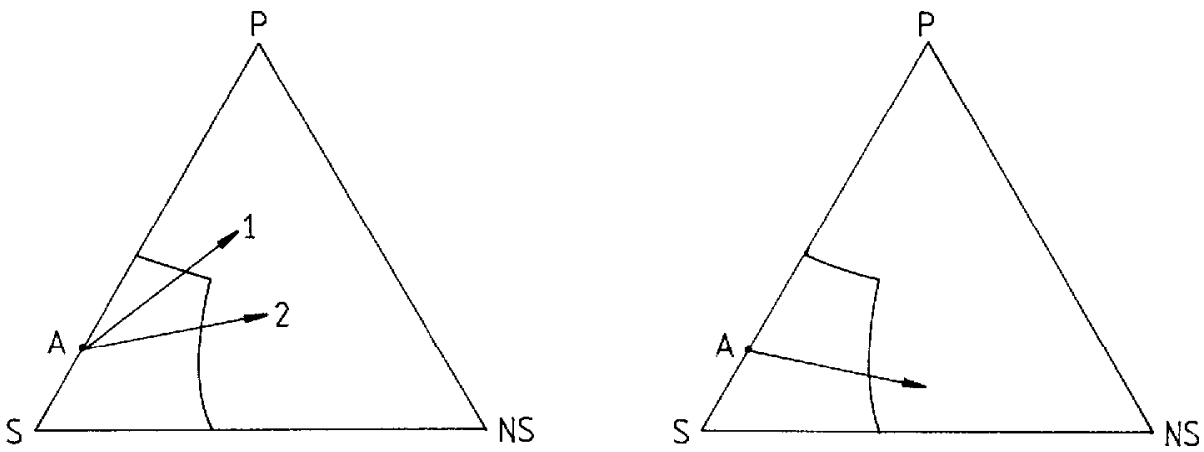

Fig. 2. Schematic course of composition for skinlayer (1) and bottomlayer (2) of a polymer film with initial composition A upon immersing into a nonsolvent bath.

Fig. 3. Coagulation path of a polymer film with initial composition A when brought in contact with a nonsolvent vapour phase saturated with solvent. 
From the model description presented here, we can infer that the direction of the coagulation path and thus the ratio of the nonsolvent inflow and solvent outflow is of the utmost importance for the resulting membrane structure.

\section{Experimental}

For membrane preparation the polymer solutions were handcast $(0.15 \mathrm{~mm})$ on a glass plate and immersed in a nonsolvent bath containing different amounts of solvent. The temperature of the coagulation bath was about $23^{\circ} \mathrm{C}$. After 30 minutes in this first bath, the membranes were transferred to a pure water bath. The structure of the membranes was studied with aid of a Jeol JSM U3 electron microscope. Micrographs were taken from the topside, the bottomside and the cross-section of the membrane. The toplayers of the membranes were examined for the presence of pores. The membranes were considered to be microporous if, at a magnification of 3000 times, pores could be detected in the toplayer (i.e. pore radius $>0.1 \mu \mathrm{m}$ ).

The membranes were made from a variety of polymer/solvent/nonsolvent systems. The nonsolvent used was water in all cases (demineralized and ultrafiltrated). The polymer-solvent pairs are listed in Table 1 . The solvents were of reagent grade and were used without further purification; the cellulose acetate (CA) was obtained from Eastman Kodak (E 398-3) and the polysulfone (PSf) was the $\mathrm{P} 3500$ type from Union Carbide. The polyvinylpyrrolidone (PVP), used as an additive to the PSf solutions, had a molecular weight of 360,000 dalton and was purchased from Fluka AG.

\section{TABLE 1}

Polymer-solvent pairs used in the experiments

\begin{tabular}{llll}
\hline Polymer & Solvent & Polymer & Solvent \\
\hline CA & DMSO & PSf & DMAc \\
CA & DMAc & PSf & NMP \\
CA & dioxane & & \\
CA & acetone & & \\
\hline
\end{tabular}

\section{Results and discussion}

\section{Microporous membranes obtained by immersion precipitation}

The addition of solvent to the coagulation bath slows down the rate of precipitation: it takes more time before the cast film becomes turbid. The same observations have been made by Strathmann [12]. At too high solvent concentrations, precipitation does not take place and the polymer slowly dissolves in the coagulation bath. 
In all the cases which we studied, see Table 1 , it appears that it is possible to produce microporous membranes by adding solvent to the coagulation bath, each system having its own minimum solvent concentration (see Table 2) required for a porous toplayer. In Fig. 4 micrographs of porous structures in the toplayer are given.

It seems that this method to obtain microporous membranes can be applied to every membrane-forming ternary system. For instance, we also know from preliminary experiments in our laboratory that the systems polyvinyllidenefluoride/ $N$-methylpyrrolidone/water and poly-2,6-dimethyl-1,4-phenylene oxide/trichloroethylene/methanol yield a microporous structure if sufficient solvent is added to the coagulation balh. Further, it is interesting to focus the attention on the paper published by Strathmann [12] on the Nomex/DMAc/ water system, and to look at the micrographs on p. 199 and the figure that displays the 'time to turbidity' as a function of the DMAc content of the coagulation bath on p. 191. In our opinion the membrane precipitated in a bath containing $75 \%$ DMAc could very well be a microporous one.

\section{TABLE 2}

Minimum solvent concentrations (\% by wt.) in the coagulation bath, if microporous toplayers are desired

\begin{tabular}{lllll}
\hline \multicolumn{2}{l}{ Casting solution } & & $\begin{array}{l}\text { Minimum solvent } \\
\text { content }(\%) \text { in } \\
\text { coagulation bath }\end{array}$ & $\begin{array}{l}\text { \%onsolvent at liquid-liquid } \\
\text { boundary in phase diagram }\end{array}$ \\
\hline Polymer & Solvent & Pol. (\%) & \\
\hline PSf & DMAc & 15 & 80 & 4 \\
PSf & NMP & 15 & 65 & 9 \\
CA & DMSO & 15 & 60 & 13 \\
CA & DMAc & 20 & 55 & 15 \\
CA & dioxane & 20 & 40 & 30 \\
CA & acetone & 20 & 30 & 28 \\
\hline
\end{tabular}

${ }^{a}$ Obtained from turbidity measurements.

Figure 4 shows the effect of an increasing solvent concentralion on the toplayer of the membranes made from a casting solution of CA in dioxane. The radius of the pores gradually increases from $0.2 \mu \mathrm{m}$ to $1 \mu \mathrm{m}$ when the solvent content increases from 30 to $50 \%$ by weight.

A well known method to increase the pore radii in the porous substructure of a polysulfone asymmetric membrane is to add a high molecular weight component to the casting solution [13]. Figure 5 shows the topsurface and the bottomsurface of a membrane precipitated from a PSf/PVP/DMAc casting solution in a mixture of $11 \%$ by weight of water in DMAc. Upon increasing the water content to $16 \%$, the pores in both the topsurface and bottomsurface become smaller, especially in the toplayer. From these micrographs it can be seen (Table 3 ) that the microporous membranes have a gradient in 

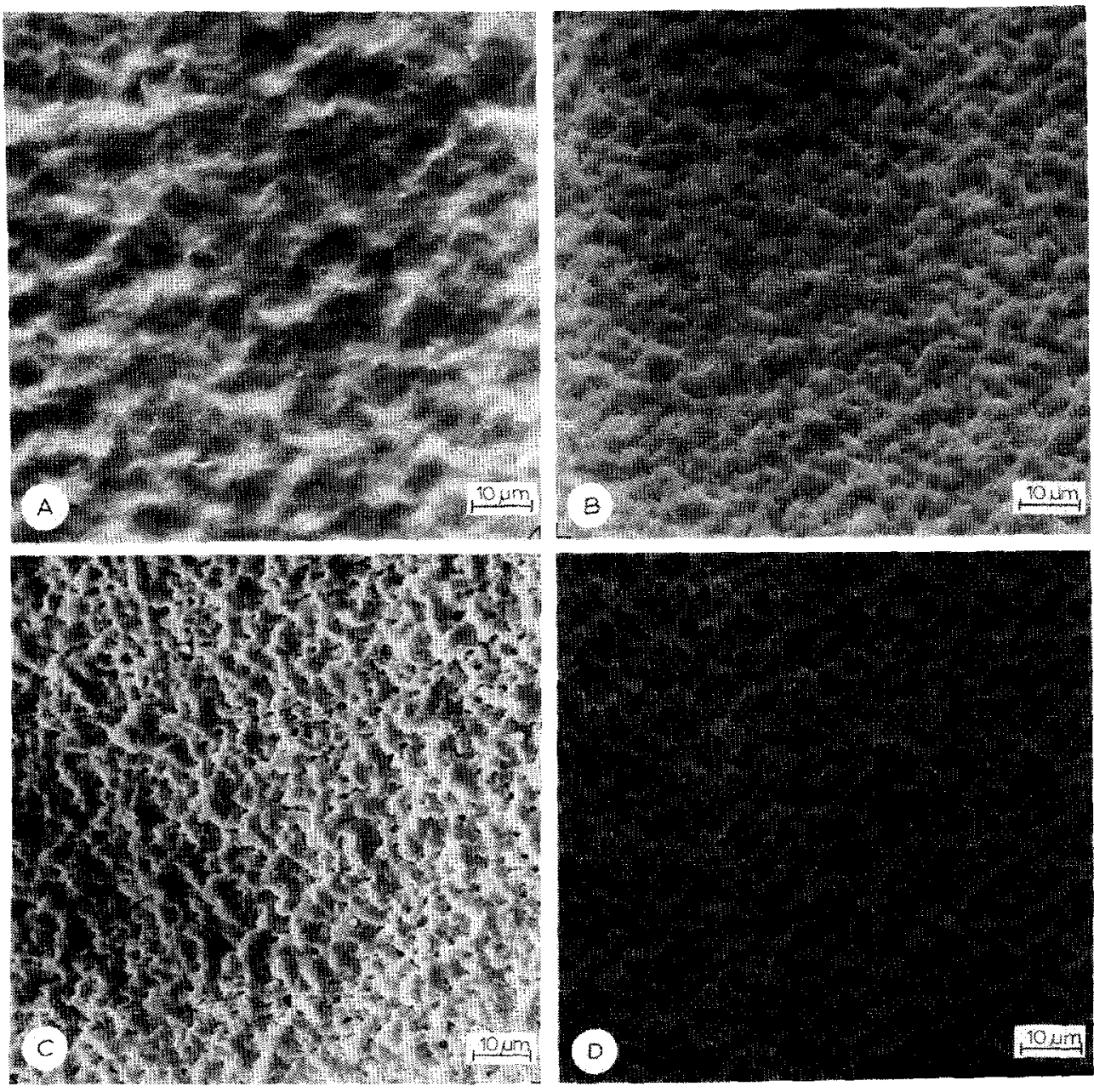

Fig. 4. Micrographs of topsurface of membranes precipitated from a casting solution of $20 \%$ by wt. CA in dioxane in a water/dioxane coagulation bath, containing (a): $30 \%,(\mathrm{~b})$ : $40 \%,(c): 45 \%$, and (d): $50 \%$ dioxane.

\section{TABLE 3}

Pore radii of microporous PSf membranes (see micrographs, Fig. 5)

$\begin{array}{lll}\text { Water (\% by wt.) } & \text { Topsurface } & \begin{array}{l}\text { Bottomsurface } \\ (\mu \mathrm{m})\end{array} \\ \text { in water/DMAc } & (\mu \mathrm{m}) & \\ \text { coagulation bath } & & \end{array}$

coagulation bath

$\begin{array}{rlr}11 & 8 & 10 \\ 16 & 0.1 & 6\end{array}$


pore radius: the pores in the toplayer are smaller than the pores in the bottomlayer.

Furthermore, it appears that changing the solvent concentration in the bath has a greater influence on the pore size in the toplayer compared to the bottomlayer. This means that with aid of two variables, i.e. the solvent concentration of the bath and the composition of the casting solution, microporous membranes with almost every combination of average pore radius and pore radius gradient can be obtained.

In this way it should be possible to make microporous films that can be used in microfiltration and in biomedical applications.
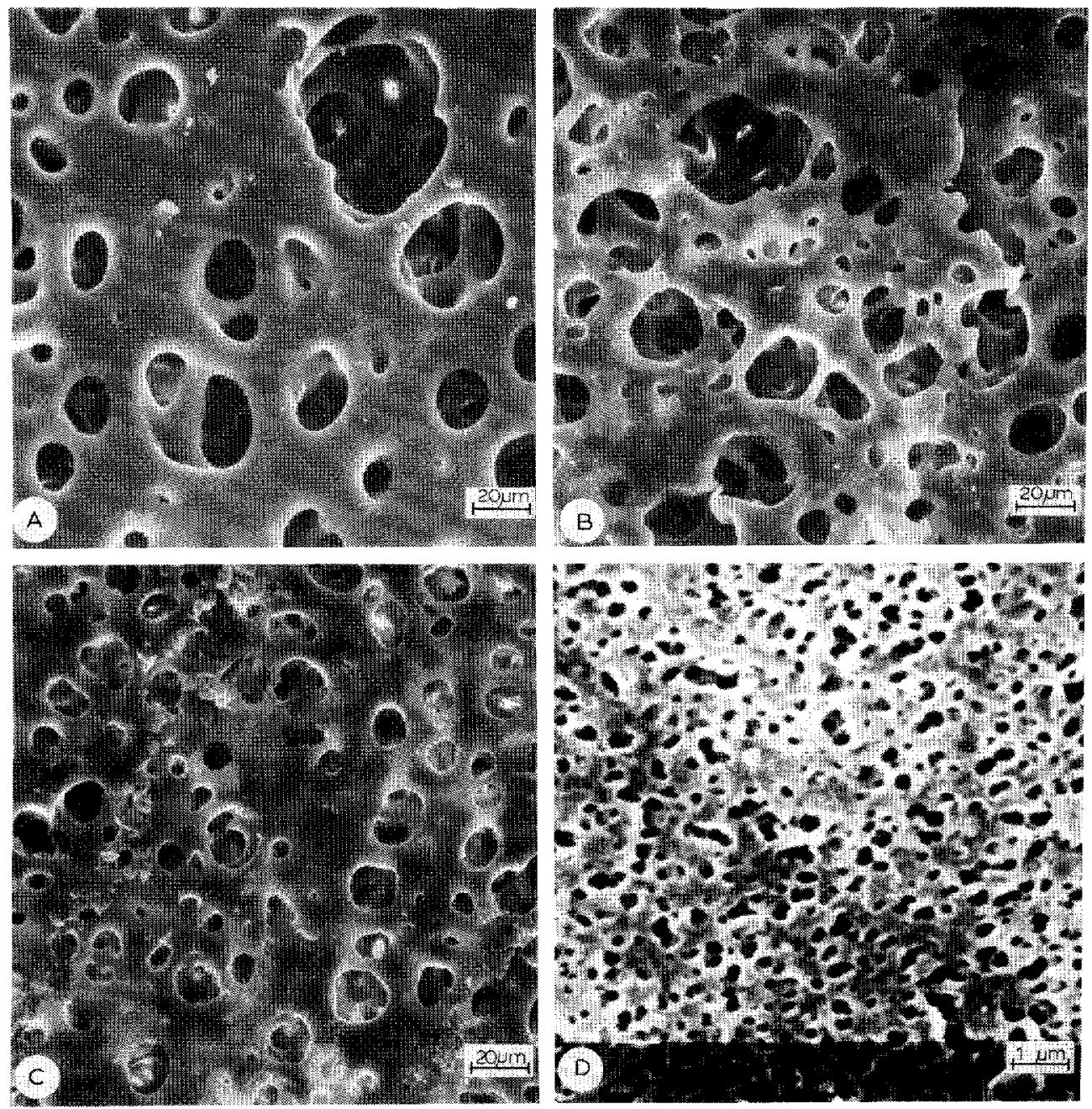

Fig. 5. Micrographs of membranes precipitated from a PSf/PVP/DMAc casting solution $(13 / 12 / 75 \%$ by wt.) in a water/DMAc coagulation bath containing $11 \%(\mathrm{a}+\mathrm{b})$ and $16 \%$ $(c+d)$ water. $a+c$ : bottom surface (glass side) $b+d$ : topsurface (bath side). 


\section{The mechanism of formation}

The interesting question is how the addition of solvent to the coagulation bath prevents the formation of a skin. To answer this, we will restrict ourselves to the ternary system.

As appeared from the model for the mechanism of formation given in the theoretical section, the ratio of solvent outflow to nonsolvent inflow is important for skin formation. We calculated the difference of chemical potential at the film/bath interface for solvent and nonsolvent as a function of solvent content in the coagulation bath (see Fig. 6). For the chemical potentials, the Flory-Huggins [14] expressions for a ternary system were used:

$$
\begin{aligned}
\frac{\Delta \mu_{1}}{R T}= & \ln v_{1}+v_{2}\left(1-\frac{\nu_{1}}{\nu_{2}}\right)+g_{12} v_{2}^{2}-v_{2}^{2} v_{1} g_{12}^{\prime}-\left[\ln \phi_{1}-\frac{\nu_{1}}{\nu_{2}} \phi_{2}\right. \\
& \left.-\frac{\nu_{1}}{\nu_{3}} \phi_{3}+\left(1-\phi_{1}\right)\left(1+g_{12} \phi_{2}+g_{13} \phi_{3}\right)-\frac{\nu_{1}}{\nu_{2}} g_{23} \phi_{2} \phi_{3}-\phi_{1} u_{2}^{2} g_{12}^{\prime}\right] \\
\frac{\Delta \mu_{2}}{R T}= & \ln v_{2}+v_{1}\left(1-\frac{\nu_{2}}{\nu_{1}}\right)+\frac{\nu_{2}}{\nu_{1}} g_{12} v_{1}^{2}+v_{1}^{2} v_{2} g_{12}^{\prime}-\left[\ln \phi_{2}-\frac{\nu_{2}}{\nu_{1}} \phi_{1}\right. \\
& \left.-\frac{\nu_{2}}{\nu_{3}} \phi_{3}+\left(1-\phi_{2}\right)\left(1+\frac{\nu_{2}}{\nu_{1}} g_{12} \phi_{2}+g_{23} \phi_{3}\right)-\frac{\nu_{2}}{\nu_{1}} \phi_{1} \phi_{3} g_{13}+\frac{\nu_{2}}{\nu_{1}} \phi_{1} u_{1} u_{2} g_{12}^{\prime}\right] \\
g_{12}^{\prime}= & \frac{\partial g_{12}}{\partial u_{2}}=\frac{\partial g_{12}}{\partial v_{2}} \\
u_{2}= & \phi_{2} /\left(\phi_{1}+\phi_{2}\right) \\
u_{1}= & \phi_{1} /\left(\phi_{1}+\phi_{2}\right)
\end{aligned}
$$

The subscripts refer to the nonsolvent (1), the solvent (2) and the polymer (3). $\Delta \mu_{i}, \phi_{i}, v_{i}$ and $v_{i}$ are the chemical potential difference over the interface,

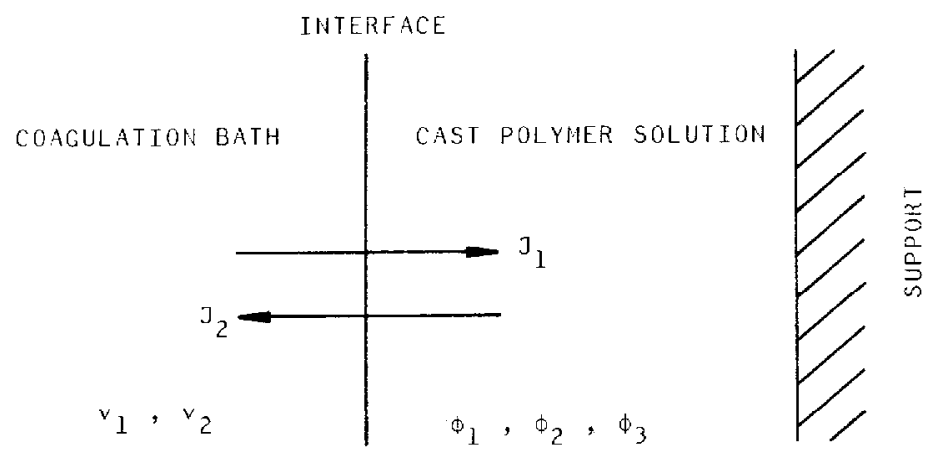

Fig. 6. Fluxes of nonsolvent $\left(J_{1}\right)$ and solvent $\left(J_{2}\right)$, respectively, at the interface of coagulation bath and cast polymer film. 
the volume fraction in the casting solution, the volume fraction in the coagulation bath and the molar volume of component $i$ respectively. $R$ is the gas constant and $T$ is the temperature in kelvin. The $g_{i j}$ parameter is the interaction parameter between components $i$ and $j ; g_{12}$ is assumed to be a function of $u_{2}=\phi_{2} /\left(\phi_{1}+\phi_{2}\right)$ in the notation of Zivný and Pouchlý [15]. Only binary interaction parameters are considered.

The interaction parameters, characteristic for the three ternary systems for which calculations have been made, are listed in Table 4 and are taken from Altena [16].

TABLE 4

Interaction parameters

\begin{tabular}{|c|c|c|c|c|c|c|c|c|c|}
\hline \multirow[t]{2}{*}{ Nonsolvent (1) } & \multirow[t]{2}{*}{ Solvent (2) } & \multirow[t]{2}{*}{ Polymer (3) } & \multirow[t]{2}{*}{$g_{13}$} & \multirow[t]{2}{*}{$g_{3,3}$} & \multicolumn{5}{|c|}{$g_{12}=a+b u_{2}+c u_{2}^{2}+d u_{2}^{3}+e u_{2}^{4}$} \\
\hline & & & & & $a$ & $b$ & $c$ & $d$ & $e$ \\
\hline water & dioxane & $\mathrm{CA}$ & 1.4 & 0.4 & 0.92 & -0.69 & 7.15 & -12.91 & 8.17 \\
\hline water & DMF & $\mathrm{CA}$ & 1.4 & 0.4 & 0.50 & 0.04 & 0.8 & -1.2 & 0.82 \\
\hline water & $\mathrm{DMF}$ & PSf & 3.7 & 0.4 & 0.50 & 0.04 & 0.8 & -1.2 & 0.82 \\
\hline
\end{tabular}

From Fig. 7 it appears that the ratio of chemical potential difference of the nonsolvent and the solvent increases upon increasing the solvent concentration in the bath. The composition taken for the casting solution is $20 \%$ by weight of polymer, $79.99 \%$ of solvent and $0.01 \%$ of nonsolvent. We have to assume that some nonsolvent is present in the casting solution, otherwise the calculated chemical potential difference will become infinite (see eqn. (1)). Since the nonsolvent concentration is very small, the calculated ratio is characteristic for the first moment of the coagulation process, i.e. the formation of the toplayer. The absolute values of $\Delta \mu_{i}$ in the various systems differ, but the ratio of $\Delta \mu_{1} / \Delta \mu_{2}$ appears to be the same. The chemical potential difference $\Delta \mu_{i}$, is the driving force for the mass transport through the film/bath interface $J_{i}$, as can be seen from the simplified phenomenological relation (cross terms neglected):

$J_{i}=L_{i}\left(\phi_{i}, v_{i}\right) \Delta \mu_{i}$

where $L_{i}$ is the permeability coefficient of component $i$, which may be a function of $\phi_{i}$ and $v_{i}$. If we disregard the influence of $L_{i}$ (on which no data are available), then the outcome of the calculation on $\Delta \mu_{1} / \Delta \mu_{2}$ (see Fig. 7) indicates that the nonsolvent inflow increases relatively to the solvent outflow if the solvent content of the bath is increased. Or, in other words, the nonsolvent inflow decreases less rapidly compared to the decreasing solvent outflow, as it is obvious that mass transfer is reduced by adding solvent to the bath.

In our opinion this means that conditions are created such that demixing 
CASTING SOLUTION : $\quad 20.00 \%$ POLYMER

$79.99 \%$ SOLVENT

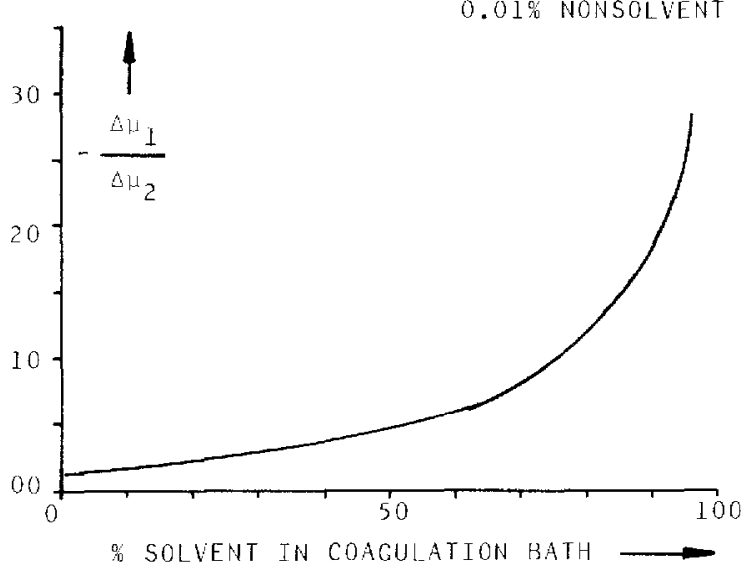

Fig. 7. The ratio $-\Delta \mu_{1} / \Delta \mu_{2}$ as a function of solvent concentration by weight in the coagulation bath; calculated for the systems CA/dioxane/water, CA/DMF/water and PSf/DMF/water.

in the toplayer will take place at lower polymer concentrations and at higher nonsolvent concentrations. As a consequence, at a certain solvent concentration in the bath, liquid-liquid phase separation will be possible in the toplayer, and the result is a toplayer with pores.

In Table 2, experimental data on some microporous membranes are gathered. In the last column, the ternary systems are characterized by the approximate water concentrations needed for liquid-liquid phase separation. It appears that systems for which a small amount of water is enough to bring about liquid-liquid phase separation need a high solvent concentration in the bath (see column 4 of Table 2 ) if microporous membranes are desired. At first sight this result may seem strange since it should be easy in such systems to reach the liquid-liquid demixing gap, as is illustrated in Fig. 8. However, it is known that the systems which are sensitive to water show a rapid coagulation with a high solvent outflow [17]. Apparently a high solvent
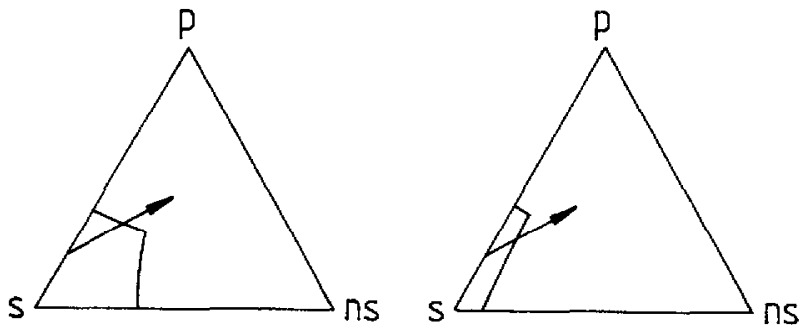

Fig. 8. Influence of the position of the liquid-liquid demixing gap on the type of phase separation at a fixed coagulation path. 
concentration in the bath is needed in those cases to decrease the solvent outflow to a sufficiently low value in order to prevent skin formation.

To give a quantitive explanation, one needs data on solvent outflow and nonsolvent inflow during coagulation for the different systems. Research on this subject is being carried out in our laboratory; the results will be published in the future.

\section{Conclusions}

Casting solutions, which immersed in pure nonsolvent give asymmetric ultraor hyperfiltration membranes, will yield microporous membranes if sufficient solvent is added to the coagulation bath.

This phenomenon can be explained with the aid of the mechanism of formation of asymmetric membranes which has been put forward in our laboratory. This model predicts that the relative magnitude of nonsolvent inflow and solvent outflow during the coagulation process has a strong influence on the ultimate membrane structure.

\section{Acknowledgement}

The authors thank F.W. Altena for the enlightening discussions on the subject.

A part of this work was made possible by a grant from the Ministerie van Economische Zaken of The Netherlands to a co-operative research project in which Wafilin B.V. and the Twente University of Technology participated. The authors wish to thank Wafilin B.V. for their permission to publish these results.

\section{References}

1 J.P.B. Baaij, patent applied for by Wafilin B.V., Hardenberg, The Netherlands (1981).

2 M.A. Frommer, I. Feiner, O. Kedem and R. Bloch, The mechanism for formation of "skinned" membranes, II. Equilibrium properties and osmotic flows determining membrane structure, Desalination, 7 (1970) 393.

3 H. Strathmann, P. Scheible and R.W. Baker, A rationale for the preparation of LoebSourirajan cellulose acetate membranes, J. Appl. Polym. Sci., 15 (1971) 811.

$4 \mathrm{H}$. Strathmann and K. Kock, The formation mechanism of phase-inversion membranes, Desalination, 21 (1977) 241.

5 M. Guillotin, C. Lemoyne, C. Noel and L. Monnerie, Physicochemical processes occurring during the formation of cellulose diacetate membranes. Research of criteria for optimizing membrane performance. IV. Ccllulose diacetate-acetone organic additive casting solutions, Desalination, 21 (1977) 165

6 D.M. Koenhen, M.H.V. Mulder and C.A. Smolders, Phase separation phenomena during the formation of asymmetric membranes, J. Appl. Polym. Sci., 21 (1977) 199.

7 L. Broens, D.M. Koenhen and C.A. Smolders, On the formation mechanism of asymmetric ultra- and hyperfiltration membranes, Desalination, 22 (1977) 205.

8 C. Cohen, G.B. Tanny and S. Prager, Diffusion-controlled formation of porous structures in ternary polymer systems, J. Polym. Sci., A-2, 17 (1979) 477. 
9 H. Bokhorst, F.W. Altena and C.A. Smolders, Formation of asymmetric cellulose acetate membranes, Desalination, 38 (1981) 349.

10 L. Broens, F.W. Altena, C.A. Smolders and D.M. Koenhen, Asymmetric membrane structure as a result of phase separation, Desalination, 32 (1980) 33.

11 F.W. Altena and C.A. Smolders, Phase separation phenomena in solutions of cellulose acetate, I. Scanning calorimetry of cellulose acetate in mixtures of dioxane and water, J. Polym. Sci. Polym. Symp., 69 (1981) 1.

12 H. Strathmann, K. Kock, P. Amar and R.W. Baker, The formation mechanism of asymmetric membranes, Desalination, 16 (1975) 179.

13 I. Cabasso, E. Klein and J.K. Smith, Polysulfone hollow fibers. II. Morphology, J. Appl. Polym. Sci., 21 (1977) 165.

14 P.J. Flory, Principles of Polymer Chemistry, Cornell Univ. Press, New York, 1953.

15 A. Zivný and J. Pouchlý, Theoretical analysis of sorption of a binary solvent in a polymer phase. I. Occurrence and character of inversion in preferential sorption, $J$. Polym. Sci., A-2, 10 (1972) 1467.

16 F.W. Altena and C.A. Smolders, Calculation of liquid-liquid phase separation in a ternary system of a polymer in a mixture of a solvent and a nonsolvent, Macromolecules, 15 (1982) 1491.

17 M.A. Frommer and D. Lancet, The mechanism of membrane formation: Membrane structures and their relation to preparation conditions, in: H.K. Lonsdale and H.E. Podall (Eds.), Reverse Osmosis Membrane Research, Plenum Press, New York, 1972. 